\title{
Economic Feasibility of Rare Earth Element Extraction from Wyoming Coal Ash/Char
}

\author{
A. J. Enriquez ${ }^{1}$, D. C. Finnoff ${ }^{1}$, \\ J. F. McLaughlin², D. A. Bagdonas ${ }^{2}$
}

Department of Economics and Finance, University of Wyoming ${ }^{1}$

Carbon Management Institute, Energy Innovation Center, Dept. 4902, University of Wyoming ${ }^{2}$

September 27, 2016 


\section{Background}

- Wyoming: largest producer of coal in U.S. ${ }^{1}$

- Coal on the decline

- Market effects

- Regulatory changes ${ }^{2}$

- Diversification $\Rightarrow$ REE extraction from coal ash?

- Increased global demand

- China dominates market
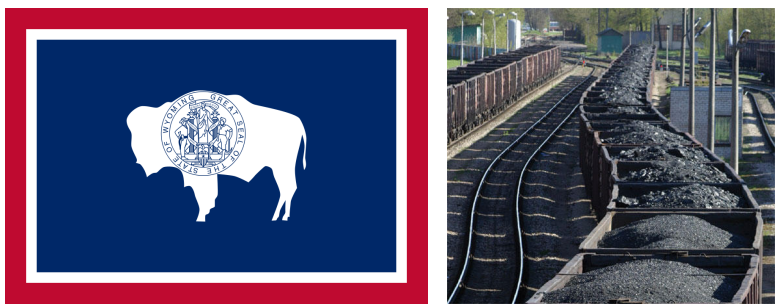

${ }^{1}(E I A, 2016)$

${ }^{2}$ (Godby et al., 2015) 


\section{REE Extraction Potential}

\section{- Taggart et al. (2016) sampled 3 ash sources:}

- Appalachian

- Illinois

- Powder River Basin (PRB)

- Results:

- PRB: lowest average total REE content

- PRB: highest extractable REE content

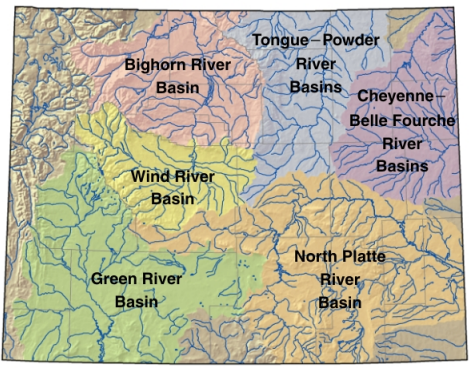




\section{Objective}

Analyze economic feasibility of RE extraction from coal ash through two economic models:

- Open-pit RE mine

- Coal stations

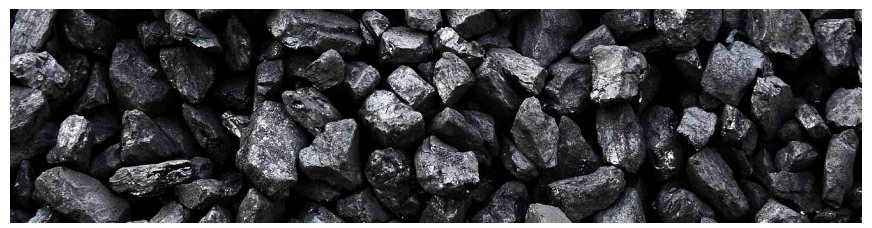




\section{Open-Pit Mine Overview}

- Small-tonnage RE mine built from scratch

- Significant start-up costs

- Capital cost of mining

- Capital cost of refining

- Estimates from the literature:

- Camm, 1991

- MIT, "Opening new mines" study

- MIT, "Green refinement" study

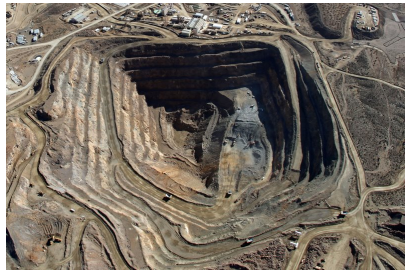

- SRK Consulting's Mountain Pass Report

- Mine-to-oxide operating cost: 1.17 US $\$$ per Ib TREO 


\section{Open-Pit Mine Results}

Initial Mining Capital Cost $\approx \$ 127$ million

Initial Refining Capital Cost $\approx \$ 100$ million

Annual Mining Operating Cost $\approx \$ 5.5$ million

Annual Refining Operating Cost $\approx \$ 387$ million

Annual Revenue $\approx \$ 265$ million

$\Rightarrow$ Large, negative net present value (NPV)

$\approx-\$ 1.9$ billion 


\section{Coal Stations Overview}

- Powder River Basin (PRB)

- Laramie River

- Dave Johnston

- WyoDak

- Dry Fork

- Green River Basin (GRB)

- Jim Bridger

- Naughton

- Data on RE concentrations in coal ash (in ppm) ${ }^{3}$

- FA, BA, and FA+BA (LA) 


\section{Model Setup: Revenue}

- Ash sources:

- Ash generated daily (rate)

- Existing landfill (stock)

- Rate Ash Calculations

- $\mathrm{ppm} \Rightarrow \%$ concentration $\Rightarrow$ multiplied by ash production rate $\Rightarrow$ converted to oxide volume

- Stock Ash Calculations

- Landfill ash completely refined by last year of operation

- Same conversion to oxide form

Volume per year $=$ rate per year + fraction of stock refined per year 


\section{TREO Volumes}

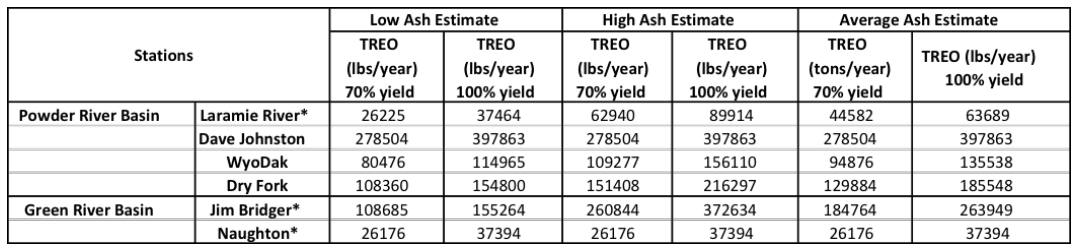

\section{Table 1: Yearly TREO Volumes}




\section{Model Setup: Revenue}

- Obtained average prices of REs using:

- Argus Media Service MetalPrices.com

- USGS Rare Earths Minerals Yearbook

- Multiplied volume per year by average price

- Summed revenue of all REs

- OMITTED EXCESSIVE RES

- Used $70 \%$ recovery rate 4

- Heated nitric acid digestion

- Assuming $95 \%$ of product is sold

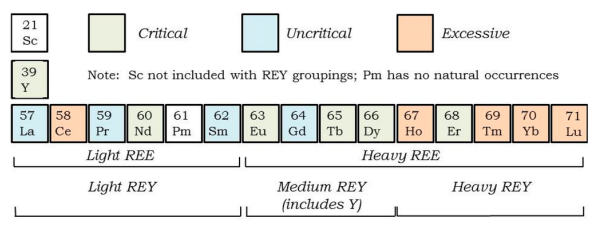

${ }^{4}$ as found by Taggart et al. when testing PRB ash 


\section{Coal Station Revenues}

\begin{tabular}{|c|c|c|c|c|c|c|c|}
\hline \multirow{2}{*}{\multicolumn{2}{|c|}{ Stations }} & \multicolumn{2}{|c|}{ Low Ash Estimate } & \multicolumn{2}{|c|}{ High Ash Estimate } & \multicolumn{2}{|c|}{ Average Ash Estimate } \\
\hline & & $\begin{array}{c}\text { Annual Revenue } \\
\text { (\$/year) } 70 \% \text { Yield }\end{array}$ & $\begin{array}{l}\text { Annual Revenue } \\
\text { Critical REE Only } \\
\text { (\$/year) } 70 \% \text { Yield }\end{array}$ & $\begin{array}{l}\text { Annual Revenue } \\
\text { (\$/year) } 70 \% \text { Yield }\end{array}$ & $\begin{array}{l}\text { Annual Revenue, } \\
\text { Critical REE Only } \\
\text { (\$/year) } 70 \% \text { Yield }\end{array}$ & $\begin{array}{l}\text { Annual Revenue } \\
\text { (\$/year) } 70 \% \text { Yield }\end{array}$ & $\begin{array}{l}\text { Annual Revenue, } \\
\text { Critical REE Only } \\
\text { (\$/year) } 70 \% \text { Yield }\end{array}$ \\
\hline \multirow[t]{4}{*}{ Powder River Basin } & Laramie River* & 889,710 & 651,846 & $2,135,303$ & $1,564,431$ & $1,512,506$ & $1,108,139$ \\
\hline & Dave Johnston & $2,475,429$ & $1,808,298$ & $2,475,429$ & $1,808,298$ & $2,475,429$ & $1,808,298$ \\
\hline & WyoDak & $1,296,431$ & 955,055 & $2,273,576$ & $1,677,264$ & $1,785,004$ & $1,316,159$ \\
\hline & Dry Fork & $1,123,246$ & 828,597 & $2,620,777$ & $1,933,426$ & $1,872,011$ & $1,381,012$ \\
\hline \multirow[t]{2}{*}{ Green River Basin } & Jim Bridger* & $3,444,956$ & $2,445,598$ & $8,267,894$ & $5,869,434$ & $5,856,425$ & $4,157,516$ \\
\hline & Naughton* & 772,678 & 562,380 & 772,678 & 562,380 & 772,678 & 562,380 \\
\hline
\end{tabular}

\section{Table 2: Yearly Revenue by Station}




\section{Model Setup: Costs}

- Cost of RE extraction from coal ash

$\Rightarrow$ largely undocumented

- Initial investment: lower

- Breakeven ash-to-oxide unit operating costs

$\Rightarrow$ using NPV equation 


\section{Model Setup: NPV}

$$
N P V_{i}=\left[\frac{1-\rho^{n+1}}{1-\rho}\right] \pi_{i t}-F C_{i}
$$




\section{Model Setup: NPV}

$$
\begin{array}{r}
N P V_{i}=\left[\frac{1-\rho^{n+1}}{1-\rho}\right] \pi_{i t}-F C_{i} \\
i=\text { station }
\end{array}
$$




\section{Model Setup: NPV}

$$
\begin{aligned}
N P V_{i}=\left[\frac{1-\rho^{n+1}}{1-\rho}\right] \pi_{i t} & -F C_{i} \\
\mathrm{i} & =\text { station } \\
\mathrm{t} & =\text { year }
\end{aligned}
$$




\section{Model Setup: NPV}

$$
\begin{aligned}
N P V_{i}=\left[\frac{1-\rho^{n+1}}{1-\rho}\right] \pi_{i t} & -F C_{i} \\
\mathrm{i} & =\text { station } \\
\mathrm{t} & =\text { year } \\
\rho & =\text { discount factor }=\frac{1}{1+r}
\end{aligned}
$$




\section{Model Setup: NPV}

$$
\begin{aligned}
N P V_{i}=\left[\frac{1-\rho^{n+1}}{1-\rho}\right] \pi_{i t}-F C_{i} \\
\qquad \mathrm{i}=\text { station } \\
\mathrm{t}=\text { year } \\
\rho=\text { discount factor }=\frac{1}{1+r} \\
\quad \quad \mathrm{r}=\text { interest rate }
\end{aligned}
$$




\section{Model Setup: NPV}

$$
\begin{aligned}
N P V_{i}=\left[\frac{1-\rho^{n+1}}{1-\rho}\right] \pi_{i t}-F C_{i} \\
\\
\mathrm{i}=\text { station } \\
\mathrm{t}=\text { year } \\
\rho=\text { discount factor }=\frac{1}{1+r} \\
\quad \quad \mathrm{r}=\text { interest rate } \\
\pi_{i t}=\text { profit }=R_{i t}-C_{i t}
\end{aligned}
$$




\section{Model Setup: NPV}

$$
\begin{aligned}
& N P V_{i}=\left[\frac{\left.1-\rho^{n+1}\right]}{1-\rho}\right] \pi_{i t}-F C_{i} \\
& \mathrm{i}=\text { station } \\
& \mathrm{t}=\text { year } \\
& \rho=\text { discount factor }=\frac{1}{1+r} \\
& \quad \mathrm{r}=\text { interest rate } \\
& \pi_{i t}=\text { profit }=R_{i t}-C_{i t} \\
& \quad R_{i t}=\text { annual revenue }
\end{aligned}
$$




\section{Model Setup: NPV}

$$
\begin{aligned}
& N P V_{i}=\left[\frac{\left.1-\rho^{n+1}\right]}{1-\rho}\right] \pi_{i t}-F C_{i} \\
& \mathrm{i}=\text { station } \\
& \mathrm{t}=\text { year } \\
& \rho=\text { discount factor }=\frac{1}{1+r} \\
& \quad \mathrm{r}=\text { interest rate } \\
& \pi_{i t}=\text { profit }=R_{i t}-C_{i t} \\
& \quad R_{i t}=\text { annual revenue } \\
& \quad C_{i t}=\text { annual cost }=w^{k} Q_{i t}^{k}
\end{aligned}
$$




\section{Model Setup: NPV}

$$
\begin{aligned}
& N P V_{i}=\left[\frac{1-\rho^{n+1}}{1-\rho}\right] \pi_{i t}-F C_{i} \\
& \mathrm{i}=\text { station } \\
& \mathrm{t}=\text { year } \\
& \rho=\text { discount factor }=\frac{1}{1+r} \\
& r=\text { interest rate } \\
& \pi_{i t}=\text { profit }=R_{i t}-C_{i t} \\
& R_{i t}=\text { annual revenue } \\
& C_{i t}=\text { annual cost }=w^{k} Q_{i t}^{k} \\
& w^{k}=\text { breakeven unit cost parameter }
\end{aligned}
$$




\section{Model Setup: NPV}

$$
\begin{aligned}
& N P V_{i}=\left[\frac{1-\rho^{n+1}}{1-\rho}\right] \pi_{i t}-F C_{i} \\
& \mathrm{i}=\text { station } \\
& \mathrm{t}=\text { year } \\
& \rho=\text { discount factor }=\frac{1}{1+r} \\
& r=\text { interest rate } \\
& \pi_{i t}=\text { profit }=R_{i t}-C_{i t} \\
& R_{i t}=\text { annual revenue } \\
& C_{i t}=\text { annual cost }=w^{k} Q_{i t}^{k} \\
& w^{k}=\text { breakeven unit cost parameter } \\
& Q_{i t}^{k}=\text { volume of ash refined }
\end{aligned}
$$




\section{Model Setup: NPV}

$$
\begin{aligned}
& N P V_{i}=\left[\frac{1-\rho^{n+1}}{1-\rho}\right] \pi_{i t}-F C_{i} \\
& \mathrm{i} \text { = station } \\
& \mathrm{t}=\text { year } \\
& \rho=\text { discount factor }=\frac{1}{1+r} \\
& r=\text { interest rate } \\
& \pi_{i t}=\text { profit }=R_{i t}-C_{i t} \\
& R_{i t}=\text { annual revenue } \\
& C_{i t}=\text { annual cost }=w^{k} Q_{i t}^{k} \\
& w^{k}=\text { breakeven unit cost parameter } \\
& Q_{i t}^{k}=\text { volume of ash refined }
\end{aligned}
$$

$F C_{i}=$ initial investment costs 


\section{Maximum Initial Investment}

Assuming absence of operating costs:

$$
F C_{i}^{\max }=\left[\frac{1-\rho^{n+1}}{1-\rho}\right] R_{i t} .
$$

- Choose level of investment below maximum

$\Rightarrow$ allows for operating costs

- Value set at $\$ 15$ million $^{5}$ 


\section{Maximum Initial Investment}

\begin{tabular}{|c|c|c|c|c|c|c|c|}
\hline \multicolumn{2}{|c|}{ Comparison of Coal Stations } & \multicolumn{2}{|c|}{$\begin{array}{c}\text { Max Initial } \\
\text { Capital Cost (\$) } \\
\text { Low Ash } \\
\text { Estimate }\end{array}$} & \multicolumn{2}{|c|}{$\begin{array}{c}\text { Max Initial } \\
\text { Capital Cost (\$) } \\
\text { High Ash } \\
\text { Estimate }\end{array}$} & \multicolumn{2}{|c|}{$\begin{array}{c}\text { Max Initial } \\
\text { Capital Cost (\$) } \\
\text { Average Ash } \\
\text { Estimate }\end{array}$} \\
\hline \multirow{4}{*}{$\begin{array}{c}\text { Powder River } \\
\text { Basin }\end{array}$} & Laramie River* & $\$$ & $11,773,959$ & $\$$ & $28,257,501$ & $\$$ & $20,015,730$ \\
\hline & Dave Johnston & $\$$ & $32,758,555$ & $\$$ & $32,758,555$ & $\$$ & $32,758,555$ \\
\hline & WyoDak & $\$$ & $17,156,304$ & $\$$ & $30,087,332$ & $\$$ & $23,621,818$ \\
\hline & Dry Fork & $\$$ & $14,864,453$ & $\$$ & $34,682,013$ & $\$$ & $24,773,233$ \\
\hline \multirow{2}{*}{$\begin{array}{c}\text { Green River } \\
\text { Basin }\end{array}$} & Jim Bridger* & $\$$ & $45,588,769$ & $\$$ & $109,413,046$ & $\$$ & $77,500,907$ \\
\hline & Naughton* & $\$$ & $10,225,222$ & $\$$ & $10,225,222$ & $\$$ & $10,225,222$ \\
\hline
\end{tabular}

Table 3: Maximum Initial Investment by Station 


\section{Zooming in on the Unit Cost Parameter}

Recall:

$$
\begin{gathered}
C_{i t}=\text { annual costs }=w^{k} Q_{i t}^{k} \\
w^{k}=\text { breakeven unit cost parameter } \\
Q_{i t}=\text { volume of ash refined }
\end{gathered}
$$

To calculate the breakeven unit cost:

$$
w^{k}=\frac{R_{i t}-\frac{F C_{i}}{\left[\frac{1-\rho^{n+1}}{1-\rho}\right]}}{Q_{i t}^{k}} .
$$

2 variants:

1. Input alternative $(k=a s h)$

2. Output alternative $(k=T R E O)$ 


\section{Zooming in on the Unit Cost Parameter}

- Recall, from SRK Mountain Pass Report:

mine-to-oxide operating cost $=\$ 1.17$ per pound TREO

- Ash-to-oxide operating cost:

ash already partly refined

$\Rightarrow$ ash-to-oxide operating cost $<$ mine-to-oxide operating cost 


\section{Zooming in on the Unit Cost Parameter}

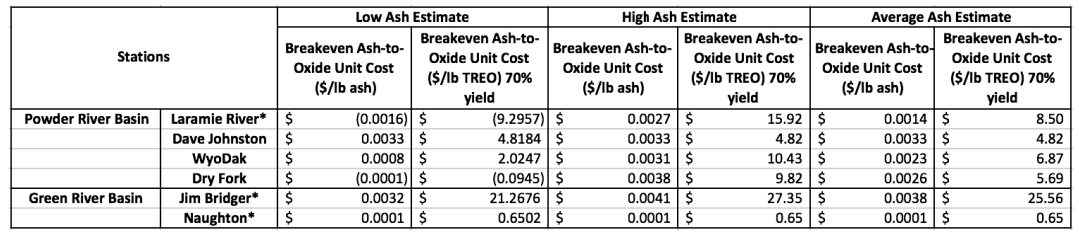

Table 4: Breakeven Unit Cost for Each Station

Notice: higher breakeven unit cost is better! 


\section{Analysis - NPV}

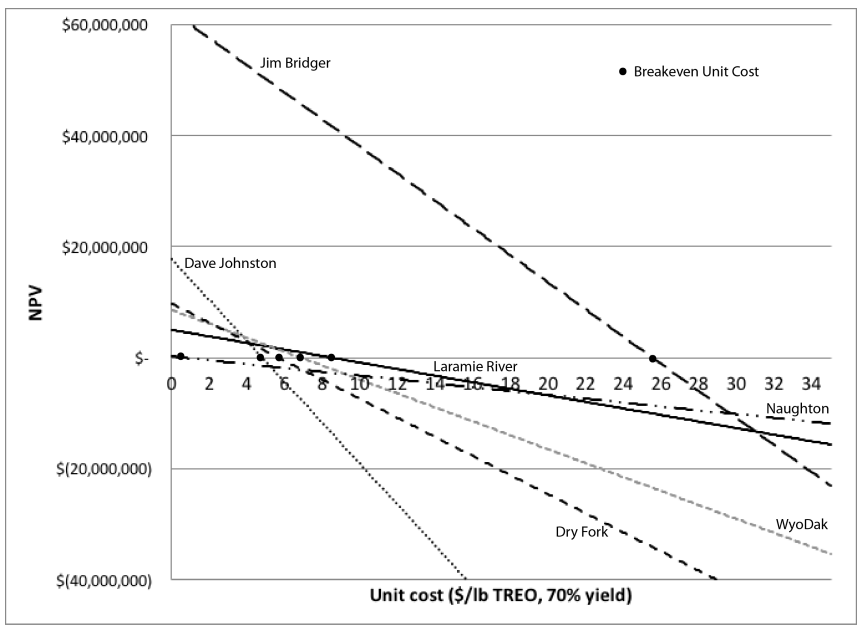

Figure 1: NPV over Unit Cost by Station 


\section{Conclusion}

- Open-pit mine:

- Building mine from ground up $\Rightarrow$ infeasible

- Refinement of REEs $\Rightarrow$ expensive

- RE extraction from coal ash:

- Lack of estimates in literature

- Model finds breakeven unit costs

$\Rightarrow$ Promising results when compared to

$\$ 1.17$ Mountain Pass value

- Big assumptions on initial capital costs 


\section{Conclusion}

- If coal stations operate under breakeven unit costs: refinement of REs from coal ash $\Rightarrow$ feasible

- Implications for Wyoming:

1. Potential source of revenue

2. Reduction in waste material

$\Rightarrow$ reduction in environmental damage
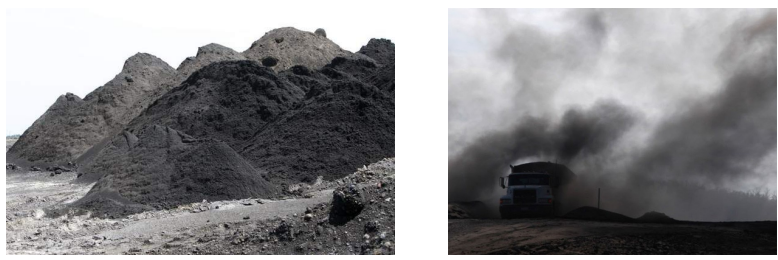


\section{Conclusion}

Questions

\section{???}
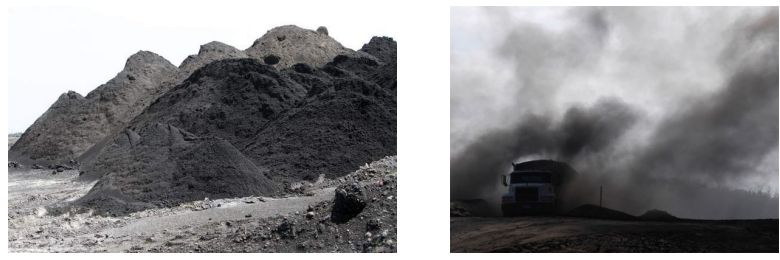


\section{References}

- Camm, T. (1991). Simplified cost models for prefeasibility mineral evaluations. Retrieved from http://pubs.usgs.gov/usbmic/ic-9298/html/cammfrms.htm.

- Godby, R., Coupal, R., Taylor, D., and Considine, T. (2015). The Impact of the Coal Economy on Wyoming. Center for Energy Economics and Public Policy, Department of Economics and Finance, University of Wyoming.

- MIT (n.d.a). Green refinement: increasing efficiency and decreasing cost and environmental damage of rare earth refinement. Retrieved from: http://web.mit.edu/12.000/www/m2016/finalwebsite/ solutions/greenrefining.html.

- MIT (n.d.b). Opening new mines: the process of mining REEs and other strategic elements. Retrieved from: http://web.mit.edu/12.000/www/m2016/finalwebsite/ solutions/newmines.html. 


\section{References (continued)}

- SRK (2010). Technical economic model for the mountain pass re-start project. Retrieved from: http://www.sec.gov/Archives/ edgar/data/1489137/000095012310065239/d74323fwfwp.htm.

- Taggart, R., Hower, J., Dwyer, G. and Hsu-Kim, H. (2016). Trends in the rare earth element content of U.S.-based coal combustion fly ashes. Environmental Science \& Technology.

- U.S. Energy Information Administration. (2016). Wyoming: State Profile and Energy Estimates. 